\title{
SOLIFENACINA: EVIDENCIA CIENTÍFICA EN EL TRATAMIENTO DE LA VEJIGA HIPERACTIVA
}

\author{
Javier Cambronero Santos y Eduardo R.Zungri Telo?.
}

Unidad de Urodinámica y Urología Funcional. Servicio de Urología. Hospital Infanta Leonor. Madrid. España. 'Consultor de Urología. Hospital POVISA. Vigo. Pontevedra. España.

\begin{abstract}
Resumen.- OBJETIVO: En este artículo se pretende analizar el uso de solifenacina en el tratamiento de la vejiga hiperactiva para objetivar su eficacia y seguridad dentro del marco de la evidencia científica.

MÉTODOS: Se ha revisado la literatura científica disponible incluyendo la mayoría de los ensayos clínicos relevantes realizados con solifenacina.

RESULTADOS: En todos los estudios analizados se aprecian mejorías significativas en los pacientes tratados con solifenacina respecto al placebo y tolterodina. Los parámetros de eficacia han sido valorados en términos objetivos y subjetivos, haciendo hincapié en la calidad de vida. El $60 \%$ de los pacientes mejoran su urgencia
\end{abstract}



(40\% no reportan urgencia), el 58\% recuperan la continencia y el $69 \%$ perciben mejoría en su condición vesical. Tras finalizar el tratamiento el $80 \%$ se encuentran satisfechos con los resultados y el $79 \%$ están dispuestos a continuarlo a largo plazo.

CONCLUSIONES: La solifenacina es eficaz en el tratamiento de la vejiga hiperactiva. La posibilidad de encontrar la dosis más eficaz, tolerable y con mejor respuesta en términos de calidad de vida supone una diferencia importante respecto a otros antimuscarínicos. El aumento a $10 \mathrm{mg}$ supone mejorías adicionales en cuanto a eficacia sin suponer un aumento significativo en los efectos adversos. Los efectos secundarios percibidos fueron leves o moderados en la mayoría de los casos, no suponiendo más abandonos de tratamiento que en el grupo de placebo. La mayoría de pacientes se sienten conformes con el tratamiento y están dispuestos a prolongarlo en el tiempo.

Palabras clave: Solifenacina. Vejiga hiperactiva. Urgencia. Antimuscarínico. Eficacia. Tolerabilidad.

Summary.- OBJECTIVES: This article aims to analyse the use of solifenacin in the treatment of overactive bladder in order to show its efficacy and safety within the framework of the scientific evidence.

METHODS: The available scientific literature was reviewed, including most of the relevant clinical trials performed with solifenacin.

RESULTS: All the studies analysed show significant improvements in patients treated with solifenacin versus placebo and tolterodine. Efficacy parameters were assessed in objective and subjective terms, with particular emphasis placed on quality of life. Urgency improves in $60 \%$ of patients (40\% report no urgency), $58 \%$ of 
patients get continence and $69 \%$ perceive an improvement in bladder condition. On completion of treatment, $80 \%$ are satisfied with the results and $79 \%$ are willing to continue with the treatment in the long term.

CONCLUSIONS: Solifenacin is efficacious in the treatment of overactive bladder. The possibility of finding the most effective and tolerable dose with the best response in terms of quality of life constitutes an important difference with regard to other antimuscarinics. The increase to $10 \mathrm{mg}$ gets additional improvements in terms of effica$c y$, without a significant increase in adverse effects. The side effects perceived were mild or moderate in most cases, and did not lead to more withdrawals compared to placebo. Most patients were satisfied with the treatment and are willing to stay on it in the long term.

Keywords: Solifenacin. Overactive bladder. Urgency. Antimuscarinic. Efficacy. Tolerability.

\section{INTRODUCCIÓN}

El síndrome de Vejiga Hiperactiva (VH) es una condición médica definida por la ICS (International Continence Society) en la actualidad como la aparición de urgencia miccional, con o sin incontinencia de urgencia, que puede ir acompañada de frecuencia y/o nocturia, siempre que se hayan descartado otras patologías del tracto urinario concomitantes como infección, litiasis, etc $(1,2)$. Esta definición aparece en 2002 resaltando la importancia de la urgencia como el síntoma principal y único indispensable para el diagnóstico.

La urgencia es el síntoma clave, definido por la incapacidad de demorar la micción. Se considera que es el síntoma "gatillo" que desencadena la aparición de los otros, es decir, si logramos controlarlo, secundariamente mejoraremos el resto de síntomas englobados en el síndrome, y el objetivo terapéutico siempre será controlar la urgencia.

Existen una serie de síntomas y patologías asociados a la $\mathrm{VH}$ con un gran impacto sobre la calidad de vida, como vergüenza, frustración, ansiedad, depresión, disfunción sexual, alteración de la afectividad, alteración en la percepción de la autoimagen, insomnio, infección urinaria y cutánea, entre otros. Los pacientes programan su vida alrededor del acto miccional, adaptando su vida a esta condición y construyen conductas de evitación (3). Además algunas personas disminuyen o paralizan su vida social, laboral y su actividad física dejando de realizar actos con los que disfrutaban, lo que lleva a una sensación de aislamiento y estrés.
La prevalencia de esta patología es extremadamente alta y aumenta con la edad. En el estudio EPIC (4) se observó una prevalencia en una población adulta de 19.165 sujetos estudiada en 5 países europeos del $19,1 \%$ en los varones y $18,3 \%$ en las mujeres mayores de 60 años, cifras mantenidas en otros estudios $(5,6)$. La frecuencia urinaria es el síntoma más frecuentemente padecido hasta en el $85 \%$ de los sujetos como se vio en un estudio poblacional en el que participaron 6 países europeos, entre ellos España, donde éste era el síntoma principal en la definición de $\mathrm{VH}$ en su contexto temporal, mientras que el $54 \%$ presentaban urgencia y el $36 \%$ incontinencia de urgencia (7). En el estudio americano NOBLE, donde participaron 5.204 adultos mayores de 18 años, se apreció $\mathrm{VH}$ con incontinencia de urgencia asociada en el $19,1 \%$ de las mujeres y $8,2 \%$ de los varones en el rango de edad de 65-74 años (6). En este estudio la prevalencia de $\mathrm{VH}$ sin incontinencia de urgencia fue mayor en hombres que en mujeres probablemente por problemas relacionados con el tracto urinario inferior y la próstata.

El tratamiento de la $\mathrm{VH}$ suele ser de tipo conservador (cambios dietéticos, restricción de líquidos, entrenamiento vesical, fisioterapia pélvica, biofeedback, electroestimulación), farmacológico (antimuscarínicos, agonistas beta-adrenérgicos en un futuro breve) y actualmente disponemos de la inyección en detrusor de toxina botulínica o de neuromodulación de raíces sacras. Sin embargo, el tratamiento de primera línea disponible en la actualidad es el antimuscarínico.

Los costes anuales asociados a la VH en USA están estimados en más de 9.000 millones de dólares anuales, con más de 1.000 millones para el tratamiento de las infecciones urinarias asociadas $(8,9)$, sin tener en cuenta los costes por pérdidas laborales y los intangibles.

Los estudios científicos en el ámbito de la incontinencia y de los síntomas del tracto urinario inferior siempre son complejos de realizar y analizar dado que en esta esfera pueden existir mejorías por el efecto placebo hasta en el $30-40 \%$ de los casos. Igual que el concepto de $\mathrm{VH}$ y su definición han ido cambiando en el tiempo, los ensayos clínicos para demostrar eficacia en esta patología también han cambiado su diseño y sus instrumentos de medida para adaptarse a los nuevos tiempos. Hace una década la mayoría de estos estudios se centraban en la incontinencia urinaria y el número de micciones diarias, pero hoy en día le damos mayor importancia a la medida de síntomas subjetivos y autopercibidos por el paciente, así como a la calidad de vida o la satisfacción con el tratamiento entre otros. 


\section{MATERIAL Y MÉTODOS}

Hemos revisado retrospectivamente las características desde el punto de vista clínico y farmacológico de la solifenacina con toda la información científica relevante acerca de su uso en el tratamiento de la vejiga hiperactiva, formada en su mayor parte por ensayos clínicos aleatorizados y controlados con placebo. En algún caso incorporamos estudios abiertos con amplias poblaciones para contrastar eficacia y seguridad en la práctica clínica habitual. Existen estudios específicamente diseñados para investigar algún aspecto de la seguridad del fármaco o su eficacia a largo plazo. En la Figura 1 podemos apreciar el desarrollo científico del fármaco, situando históricamente los ensayos clínicos más relevantes sobre su uso.

\section{DISCUSIÓN}

\section{Farmacología}

El succinato de solifenacina es una molécula con acción bloqueadora de los receptores muscarínicos de tipo M3, y en menor medida los M2. Se ha demostrado in vitro e in vivo la mayor selectividad por la vejiga ("uroselectividad") de la solifenacina respecto a otros antimuscarínicos, siendo 40 veces menos potente que la oxibutinina y 79 veces menos que la tolterodina en la inhibición de la secreción salivar por su mayor selectividad por los receptores muscarínicos M3 (10). Su efecto inhibidor en el músculo liso vesical es 3,6 veces mayor que en las glándulas salivares (1 1 ) lo que demuestra mayor afinidad por la vejiga.

Se metaboliza mayoritariamente en el hígado por el grupo de enzimas CYP3A4. Existe un metabolito activo excretado en orina activo, que hoy día se sospecha puede tener efecto local sobre los receptores muscarínicos a nivel urotelial (12).

La vida media de eliminación es de 50 horas, lo que demuestra una cobertura suficientemente amplia para controlar los síntomas diariamente en una patología de tipo crónico.

Las únicas interacciones con fármacos significativas son con los que utilizan la misma vía de metabolización hepática, como puede ser el ketoco-

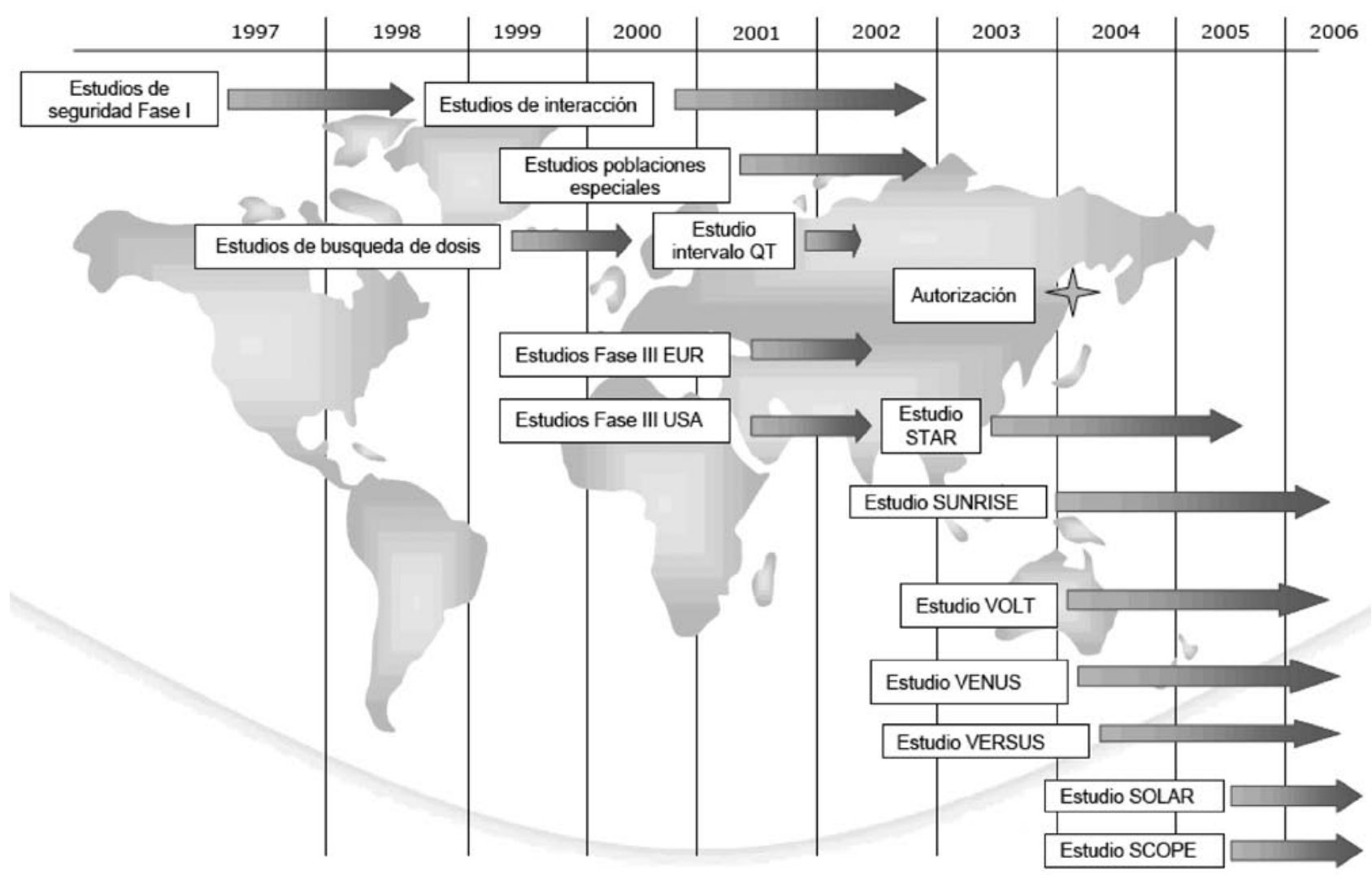

FIGURA 1. Desarrollo clínico de la Solifenacina (Adaptado de Robinson D, Cardozo L. Solifenacin: pharmacology and clinical efficacy. Exp Rev Clin Pharmacol 2009; 2(3): 239-53). 
nazol, de forma que la dosis máxima de solifenacina deberá limitarse a $5 \mathrm{mg}$ cuando se administre simultáneamente con ketoconazol u otros inhibidores de la CYP3A4. No se recomienda el uso de solifenacina en casos de insuficiencia hepática moderada e insuficiencia renal grave en tratamiento simultaneo con un inhibidor potente de la CYP3A4 (13).

\section{Seguridad}

La solifenacina ha demostrado ser el único antimuscarínico más selectivo por el músculo liso vesical que por las glándulas salivales, lo que amortigua la sequedad de boca. No se han descrito apenas efectos secundarios graves (incluyendo en la esfera cardiovascular y el sistema nervioso central). La discontinuación dentro de ensayos clínicos controlados por efectos adversos oscila entre el 0,3\% (14) y el $4,7 \%$ (15) lo que va en consonancia con lo anteriormente descrito.

La posibilidad de flexibilizar la dosis entre 5 y $10 \mathrm{mg}$ tanto para aumentar como para disminuir durante el tratamiento permite individualizar la dosis más efectiva y con menos efectos secundarios para cada paciente, requiriendo eso sí comunicación entre éste y el médico. El incremento de la dosis de 5 a 10 $\mathrm{mg}$ no produce un aumento significativo de los efectos secundarios. Menos del 3,6\% de los pacientes abandonaron precozmente el tratamiento por efectos secundarios en los estudios STAR y SUNRISE (en comparación con el $3 \%$ de los tratados con tolterodina de liberación prolongada (LP) en el estudio STAR y el $2,7 \%$ de los tratados con placebo en el estudio SUNRISE) $(16,17)$.

La "sequedad de boca" ocurre en el $85 \%$ de los pacientes con oxibutinina (15 mg/día) (18) y $21,8 \%$ con trospio (19). Con solifenacina en aproximadamente el $7,7-14 \%$ con $5 \mathrm{mg} /$ día y en el 21,3 $23,1 \%$ con $10 \mathrm{mg} /$ día según los estudios (frente a $3,5 \%$ en placebo). La mayoría de estos eventos fueron considerados por los pacientes como leves-moderados $(92 \%-100 \%)$, y las cifras de abandono por el mismo no tuvieron diferencias respecto al grupo placebo (Tabla I).

El "estreñimiento" ocurre en el $24 \%$ de los que toman oxibutinina ( $15 \mathrm{mg} /$ día) y $2,6 \%$ con tolterodina de liberación prolongada (LP) $(4 \mathrm{mg} /$ día) $(20,21)$. Con solifenacina en $5,4 \%$ con $5 \mathrm{mg} /$ día y $8,5 \%$ con $10 \mathrm{mg} /$ día (frente a 1,9\% en placebo). $(22,23,24)$. Este evento fue considerado leve-moderado por los pacientes en el $86,1 \%-100 \%$ de los casos. Las cifras de abandono de tratamiento por este efecto no tienen diferencias respecto al placebo. Realmente este efecto adverso sólo puede llegar a ser relevante en algunos ancianos que ya padezcan este síntoma previamente.
La "visión borrosa" con solifenacina ocurre en el $3,8 \%$ de pacientes con $5 \mathrm{mg} /$ día y en el $5,9 \%$ con $10 \mathrm{mg} /$ día (frente a $2,5 \%$ en placebo). $(22,23$, 24). Las cifras de abandono por este síntoma no tienen diferencias con el placebo. Fue considerada leve-moderada por más del $90 \%$ de los pacientes.

Otros efectos adversos raros descritos son somnolencia, alteración del gusto $(<1 \%)$. Se han detectado también alteraciones del intervalo QT clínicamente no significativas, sin ninguna influencia sobre los signos vitales o parámetros de laboratorio en los estudios realizados con dosis altas $(25,26)$.

En el anciano, según los estudios que poseemos los efectos adversos fueron similares a la población joven, siendo la mayoría de ellos de intensidad leve o moderada $(27,28)$.

En mujeres embarazadas y lactantes no hay estudios específicos, por lo cual no se recomienda su uso en estas situaciones.

Durante el estudio a largo plazo en fase III de Solifenacina se vio que el tratamiento a largo plazo es seguro y bien tolerado, sólo abandonaron el estudio por efectos secundarios el $4,7 \%$ de los pacientes. Durante el estudio sólo el 0,4\% abandonaron por sequedad de boca. Al final del estudio de 52 semanas el $74 \%$ de los pacientes estaban satisfechos con la eficacia del tratamiento (otro $21 \%$ refiere mejoría) y el $99 \%$ referían una tolerabilidad satisfactoria o aceptable (15).

Se diseñó un estudio en fase IV abierto para determinar la seguridad cardiovascular y la tolerabilidad de la solifenacina en el uso clínico rutinario (29). Se quería ver si este antimuscarínico tenía influencia en la frecuencia cardiaca y si podría ser peligroso en pacientes con cardiopatía isquémica o insuficiencia cardiaca. También si podría interferir con los fármacos usados frecuentemente en estas patologías. Se estudiaron durante 12 semanas 4450 pacientes en Alemania con $\mathrm{VH}$ tratados en consultas de urología. De ellos el 41,7\% tenían hipertensión arterial, el 11,9\% tenían antecedentes de enfermedad coronaria, el $1,6 \%$ habían sufrido algún infarto de miocardio, el $7 \%$ tenían diagnóstico de insuficiencia cardiaca y el $5,3 \%$ padecían alguna otra enfermedad cardiovascular. El 17,3\% entraron en el estudio presentando alguna anomalía en el ECG. Tras las 12 semanas de tratamiento en dosis flexible con $5-10 \mathrm{mg}$ de solifenacina no se detectaron cambios (pre-tratamiento vs pos-tratamiento) ni en la frecuencia cardiaca $(75,2$ vs 74,5$)$ ni en la tensión arterial (137/82 vs 134/81). En el subgrupo de alteraciones en el ECG no se vieron al finalizar el estudio un aumento signi- 
ficativo de éstas. No se apreció un mayor índice de efectos adversos en el grupo de pacientes con enfermedades cardiovasculares durante el estudio.

El estudio SCOPE (30) fue diseñado para objetivar si una dosis de $10 \mathrm{mg}$ de solifenacina frente a $10 \mathrm{mg}$ de oxibutinina en sujetos mayores de 65 años sanos producía alteraciones en la esfera cognitiva. Como sabemos en esta franja de edad la $\mathrm{VH}$ es una patología frecuente, así como los trastornos cognitivos secundarios a la edad, enfermedad cerebrovascular, demencia, Parkinson o trastornos psiquiátricos. Estos pacientes requieren con cierta frecuencia tratamiento con antimuscarínicos que podrían tener efectos secundarios de este tipo. En un grupo de 12 sujetos sanos mayores de 65 años se administró se- cuencialmente con un periodo de lavado intermedio de 14 días una dosis de solifenacina $10 \mathrm{mg}$, oxibutinina $10 \mathrm{mg}$ y placebo. Se aplicaron una batería de test psicométricos a las $2,4,6,8,10,12$ y 24 horas de su administración, para valorar aspectos como la atención, el procesamiento de información, la memoria, la alerta y el estado de humor. Tras el análisis de los datos no se apreciaron cambios significativos respecto al placebo en ninguno de estos parámetros tras la administración de solifenacina. Con Oxibutinina hubo cambios significativos frente a placebo $(p<0,05)$ en cuanto a atención, memoria y estado de alerta.

Dado que la solifenacina se metaboliza por el complejo citocromo P450(CYP)3A4, se ve afecta-

\section{TABLA I. EFECTOS ADVERSOS DETECTADOS EN EL TRATAMIENTO CON SOLIFENACINA.}

\begin{tabular}{|c|c|c|c|c|}
\hline Estudio & $\begin{array}{c}015 \\
\text { Ref. } 23\end{array}$ & $\begin{array}{c}018 \\
\text { Ref. } 24\end{array}$ & $\begin{array}{c}019 \\
\text { Ref. } 15\end{array}$ & $\begin{array}{c}\text { STAR } \\
\text { Ref. } 16\end{array}$ \\
\hline & S5 / S10 & S5 / S10 & S5 / S10 & S5-10 \\
\hline Boca seca & $14 \% / 21,3 \%$ & $7,7 \% / 23,1 \%$ & $10,2 \% / 17,4 \%$ & $30 \%$ \\
\hline$\%$ leve-moderado & ND & $100 \%$ & $96,1 \% / 92 \%$ & $94,3 \%$ \\
\hline Estreñimiento & $7,2 \% / 7,8 \%$ & $3,7 \% / 9,1 \%$ & $4,9 \% / 7,9 \%$ & $6,4 \%$ \\
\hline \% leve-moderado & ND & $100 \% / 93.4 \%$ & $93,9 \% / 86,1 \%$ & $92,2 \%$ \\
\hline Visión borrosa & $3,6 \% / 5,6 \%$ & $4 \% / 5,9 \%$ & $4,1 \% / 4,4 \%$ & $0,7 \%$ \\
\hline$\%$ leve-moderado & ND & $90 \% / 100 \%$ & $97,7 \%$ & $100 \%$ \\
\hline
\end{tabular}

\begin{tabular}{|l|c|c|c|c|}
\hline \multicolumn{1}{|c|}{ Estudio } & $\begin{array}{c}\text { SUNRISE } \\
\text { Ref. 17 }\end{array}$ & $\begin{array}{c}\text { VOLT } \\
\text { Ref. 45 }\end{array}$ & $\begin{array}{c}\text { VERSUS } \\
\text { Ref. 48 }\end{array}$ & $\begin{array}{c}\text { VIBRANT } \\
\text { Ref. 14 }\end{array}$ \\
\hline Boca seca & S5-10 & S5-10 & S5-10 & S5-10 \\
\hline$\%$ leve-moderado & $15,8 \%$ & $21,4 \%$ & $17,5 \%$ & $13,2 \%$ \\
\hline Estreñimiento & ND & $94,9 \%$ & $98,8 \%$ & $\mathrm{ND}$ \\
\hline$\%$ leve-moderado & $\mathrm{ND}$ & $13,3 \%$ & $11,6 \%$ & $8 \%$ \\
\hline Visión borrosa & $0,8 \%$ & $92,5 \%$ & $93,9 \%$ & $\mathrm{ND}$ \\
\hline$\%$ leve-moderado & $\mathrm{ND}$ & $2,6 \%$ & $2,3 \%$ & $1 \%$ \\
\hline
\end{tabular}

(S5 / S10: efectos adversos referidos según dosis; S5-10: efectos adversos con dosis flexible en conjunto; ND: datos no disponibles). 
da por el ketoconazol que es un potente inhibidor, aumentando la AUC y la Cmax (1,43 veces) (31). Se ha demostrado que se puede administrar junto a anticonceptivos anovulatorios orales sin restar eficacia a éstos (32). Se puede administrar con seguridad junto a anticoagulantes warfarínicos y digoxina no siendo preciso ningún ajuste de dosis (33). No hay que tener precaución especial al administrarse a pacientes con insuficiencia renal leve o moderada, estando indicado no sobrepasar la dosis de $5 \mathrm{mg}$ diarios cuando ésta es grave (34). En el caso de insuficiencia hepática leve no debe tenerse precaución especial. Si es moderada no se recomienda sobrepasar dosis de 5 mg diarios ya que la vida media, AUC del fármaco y sus metabolitos se pueden ver aumentados (35).

\section{Eficacia}

La eficacia de la solifenacina frente al placebo ha sido sobradamente demostrada en ensayos clínicos en fase II-III y postcomercialización publicados sobre el tema.

\section{Fase II.}

El primer estudio en fase II se desarrolló en Europa con 225 pacientes y fue controlado con tolterodina $2 \mathrm{mg}$ y placebo. La solifenacina a dosis de 5,10 y $20 \mathrm{mg}$ demostró diferencias estadísticamente significativas (DES) frente a placebo en cuanto a la frecuencia miccional y el volumen medio por micción, y fue superior al placebo respecto a la incontinencia y los episodios de urgencia en 24 horas, lo que no ocurrió con $2,5 \mathrm{mg}(36,37)$. Se vio que la mejoría de los síntomas medidos se apreciaba ya a las 2 semanas de tratamiento, y se comprobó que las dosis eficaces más seguras fueron de 5 y $10 \mathrm{mg}$.

El segundo estudio en fase II desarrollado en USA con 265 pacientes seleccionó pacientes con más de 8 micciones diarias, siendo tratados con solifenacina a dosis de 2,5-5-10-20 mg frente a placebo. De forma similar al anterior, el número de micciones y los episodios de incontinencia/24h fueron menores y el aumento del volumen vaciado por micción fue mayor en los grupos de solifenacina al final del periodo de estudio.

En estos estudios se demostró que las dosis de 5 y $10 \mathrm{mg}$ de solifenacina son las más eficaces clínicamente, consiguiendo un correcto balance entre eficacia, calidad de vida y tolerabilidad, por lo que fueron seleccionadas para futuros estudios a gran escala en fase III.

\section{FASE IIIa}

En 6 estudios a gran escala se han estudiado a 3700 pacientes con síntomas de $\mathrm{VH}$. En los 2 estudios iniciales realizados en USA ("013" y "014")
$(38,39)$ se investigó la eficacia de solifenacina 10 mg diarios en dosis única frente a placebo en 1208 pacientes de ambos sexos. En ambos se demostró con DES $(p<0,001)$ que fue superior al placebo al disminuir la frecuencia miccional (variable principal), así como los episodios de incontinencia / 24h, los episodios de urgencia / 24h, y aumentó el volumen medio de cada micción.

En el estudio "015" (23) se estudió en 1081 pacientes la eficacia de solifenacina 5 y $10 \mathrm{mg}$ usando como controles tolterodina $2 \mathrm{mg}$ y placebo, viéndose una reducción frente al placebo con DES $(p<0,001)$ en el número de episodios de urgencia/24h del $52 \%$ y $55 \%$ respectivamente en el grupo de solifenacina, así como en los episodios de incontinencia/24h 163 . $65 \%)$, en la frecuencia miccional (17-20\%) y aumento en el volumen medio por micción (25-29\%). No ocurrió así en el grupo de Tolterodina donde las diferencias no fueron significativas.

El estudio "018" (24) estudió en 1091 pacientes (907 evaluables) tratados con solifenacina 5 y $10 \mathrm{mg}$ su eficacia frente al placebo. La frecuencia miccional (variable principal) se redujo con DES $(p<0,005 / p<0,001)$ en ambas dosificaciones frente al placebo $(-2,37 /-2,81)$. También se redujeron los episodios diarios de incontinencia por urgencia y totales en ambas dosis, recuperando la continencia algo más del $50 \%$ de los pacientes tratados. La nocturia se redujo de forma significativa en el grupo de solifenacina $10 \mathrm{mg}(38,5 \% \mathrm{DES})$. Los episodios de urgencia se redujeron un $51-52 \%$ entre los tratados con solifenacina, y el volumen medio de cada micción aumentó de forma significativa $(p<0,001)$.

El estudio "019" (15) es un estudio abierto de extensión, con 52 semanas de duración que recogió el $91 \%$ de los pacientes (n: 1633) de los dos estudios europeos anteriores que quisieron continuar con el tratamiento. Todos recibieron solifenacina $5 \mathrm{mg}$ durante 4 semanas pudiendo flexibilizar la dosis a continuación. Se mantuvo la eficacia similar a la ya registrada al final de las 12 semanas de los estudios previos al final de las 40 semanas de extensión en todos los síntomas principales (reducción de los episodios de urgencia -63\%-, disminución de la frecuencia miccional -23\%-, disminución de los episodios de incontinencia $-66 \%$ - y aumento del volumen por micción $-31 \%-$. Al final del estudio el $40 \%$ de los pacientes referían no tener urgencia y el $58 \%$ eran continentes. Esto demuestra que la satisfacción del paciente a largo plazo es alta, con cifras de tolerabilidad a partir de las 24 semanas del $99 \%$ (satisfactoria -85\%, aceptable - $13 \%-$-) y una satisfacción con la eficacia percibida por el paciente del $95 \%$ a partir de las 24 semanas (74\% satisfechos, $21 \%$ con beneficios). 
TABLA II. ANÁLISIS "POST HOC" DE LA POBLACIÓN DE PACIENTES $\geq 65$ AÑOS EN LOS ESTUDIOS 015, 018 (28).

\begin{tabular}{|c|c|c|c|}
\hline & Solifenacina 5mg & Solifenacina 10mg & Placebo \\
\hline Episodios incontinencia/24h & $-1,5\left(^{*}\right)$ & $-1,9\left(^{* *}\right)$ & -1 \\
\hline Episodios urgencia/24 h & $-3,2\left(^{* *}\right)$ & $-3,2\left(^{* *}\right)$ & $-1,6$ \\
\hline No micciones/24h & $-2\left(^{* *}\right)$ & $-2,5\left(^{* *}\right)$ & $-1,1$ \\
\hline Volumen/micción (cc) & $30,2\left(^{* *}\right)$ & $46,2\left(^{* *}\right)$ & 9,1 \\
\hline$\%$ continentes & $49,1 \%\left(^{* *}\right)$ & $47,3 \%\left(^{* *}\right)$ & $28,9 \%$ \\
\hline$\%$ pacientes sin urgencia & $34,6 \%\left(^{* *}\right)$ & $24,9 \%\left(^{* * *}\right)$ & $16,9 \%$ \\
\hline
\end{tabular}

$\left.\right|^{\star}\left|p<0,05 ; 1^{* \star}\right| p<0,001 ; 1^{* \star *} \mid p<0,01$

TABLA III. RESOLUCIÓN / NORMALIZACIÓN DE LOS SÍNTOMAS DE VH (4 1).

\begin{tabular}{|l|c|c|c|c|c|c|c|c|}
\hline & \multicolumn{2}{|c|}{$\begin{array}{c}\text { Resolución } \\
\text { Urgencia }\end{array}$} & \multicolumn{2}{c|}{ Continencia } & \multicolumn{2}{c|}{$\begin{array}{c}\text { Normalización } \\
\text { Frecuencia }\end{array}$} & \multicolumn{2}{c|}{$\begin{array}{c}\text { Resolución } \\
\text { Nocturia }\end{array}$} \\
\hline & $\%$ & DES & $\%$ & DES & $\%$ & DES & $\%$ & DES \\
\hline Placebo & 15,5 & & 34,1 & & 22,4 & & 14,5 & \\
\hline Solifenacina 5 $\mathbf{~ m g . ~}$ & 28,6 & $\mathrm{P}<0,001$ & 50,6 & $\mathrm{P}<0,001$ & 33 & $\mathrm{P}<0,001$ & 16,2 & \\
\hline Solifenacina 10 $\mathbf{~ m g . ~}$ & 25,5 & $\mathrm{P}<0,001$ & 51,8 & $\mathrm{P}<0,001$ & 37,3 & $\mathrm{P}<0,001$ & 19 & $\mathrm{P}<0,01$ \\
\hline
\end{tabular}

TABLA IV. PACIENTES CON UNA REDUCCIÓN >50\% DE SUS SÍNTOMAS DE VH (4 1).

\begin{tabular}{|l|c|c|c|c|c|c|c|c|}
\hline & \multicolumn{2}{|c|}{$\begin{array}{c}\text { Episodios } \\
\text { Urgencia /24h }\end{array}$} & \multicolumn{2}{c|}{$\begin{array}{c}\text { Episodios } \\
\text { Incontinencia /24h }\end{array}$} & \multicolumn{2}{c|}{$\begin{array}{c}\text { Número } \\
\text { Micciones /24h }\end{array}$} & \multicolumn{2}{c|}{$\begin{array}{c}\text { Episodios } \\
\text { Nocturia /24h }\end{array}$} \\
\hline & $\%$ & DES & $\%$ & DES & $\%$ & DES & $\%$ & DES \\
\hline Placebo & 43,8 & & 57,6 & & 43,5 & & 36,2 & \\
\hline Solifenacina 5 mg. & 61,9 & $\mathrm{P}<0,001$ & 70,7 & $\mathrm{P}<0,001$ & 60,5 & $\mathrm{P}<0,001$ & 43,3 & $\mathrm{P}<0,01$ \\
\hline Solifenacina 10 $\mathbf{~ m g . ~}$ & 66,2 & $\mathrm{P}<0,001$ & 78,5 & $\mathrm{P}<0,001$ & 63,6 & $\mathrm{P}<0,001$ & 43,6 & $\mathrm{P}<0,001$ \\
\hline
\end{tabular}

En el análisis "post hoc" de la población de pacientes mayores de 65 años participantes en los estudios 015,018 y 019 (28) podemos ver apiladas las cifras de eficacia con solifenacina 5 y $10 \mathrm{mg}$. (Tabla II).

Con estos estudios apreciamos que el tratamiento con solifenacina es eficaz para el control de los episodios de urgencia, síntoma central del síndro- me de $\mathrm{VH}$, reduciéndolos de forma significativa en más del $60 \%$ de los pacientes (desapareciendo en alrededor del $40 \%$ ), y secundariamente disminuyendo el resto de síntomas relacionados del síndrome $(15,23,24,40)$. Existe una revisión de 4 ensayos clínicos en fase III (41) donde se analizan en conjunto los resultados con 2800 pacientes en tratamiento con dosis de 5 y $10 \mathrm{mg}$. (Tablas III y IV). Al reducir la urgencia miccional conseguimos aumentar el "warning 
time" (tiempo desde la primera sensación de urgencia hasta el vaciado, sea voluntario o involuntario), que es necesario para la replección vesical, aumentando la capacidad funcional de ésta y el volumen de cada micción, y consecuentemente reduciendo la frecuencia miccional y la incontinencia.

\section{FASE IIIb}

En el estudio europeo STAR (16) se randomizaron 1200 pacientes con vejiga hiperactiva que presentaban ocho o más micciones diarias con uno o más episodios de urgencia/24h y/o incontinencia/ $24 \mathrm{~h}$ en los 3 días previos. Los pacientes pasaron a tomar $5 \mathrm{mg}$ de solifenacina o $4 \mathrm{mg}$ de tolterodina LP, pudiendo aumentar la dosis de solifenacina a $10 \mathrm{mg}$ a las 4 semanas. Al analizar la disminución del número de micciones en 24 horas (variable principal), la dosis flexible de solifenacina no fue inferior $(-2,45$ vs $-2,24)$ a la de tolterodina LP a las 12 semanas de tratamiento ( $p=0,004$ para no inferioridad). El grupo de pacientes con dosis flexible de solifenacina tuvo una mejoría con DES respecto al grupo tolterodina en el resto de variables estudiadas (episodios de urgencia e incontinencia/24h, autopercepción de la condición vesical, volumen miccional, uso de absorbentes, reducción del $50 \%$ de los episodios de incontinencia y del volumen vaciado). El 59\% de los pacientes con incontinencia tratados con solifenacina se volvieron continentes. El $48 \%$ de los pacientes que tomaron solifenacina solicitaron un aumento de la dosis después de 4 semanas. (Tabla V).

De aquí en adelante se abre una nueva etapa en el diseño de ensayos clínicos basado en el cambio conceptual de la definición de VH. La mayoría de estudios diseñados hasta el momento se centraron en la medición de variables miccionales objetivables en los diarios miccionales. Estas ayudan a cuantificar los síntomas objetivos, pero perdemos la información que nos pueden aportar los pacientes en cuanto a síntomas subjetivos que incluso en la propia vivencia del paciente pueden reflejar de manera más directa o fiable los cambios experimentados tras la introducción de un nuevo tratamiento. Es importante explorar la percepción del paciente sobre el impacto que tiene la $\mathrm{VH}$ sobre su vida diaria y la mejoría subjetiva que aprecia tras un tratamiento. Hoy día es ampliamente aceptado que cualquier intervención médica debe resultar en una mejoría de la calidad de vida similar a la eficacia clínica demostrada, siendo esto particularmente importante en las enfermedades crónicas como es el caso de la VH. Dado que la percepción de los

TABLA V. PARÁMETROS DE EFICACIA EN EL ESTUDIO STAR (16).

\begin{tabular}{|c|c|c|}
\hline & $\begin{array}{l}\text { Solifenacina dosis flexible } \\
\qquad 5-10 \mathrm{mg}\end{array}$ & $\begin{array}{c}\text { Tolterodina LP } \\
4 \mathrm{mg}\end{array}$ \\
\hline № Micciones/24h ( $p=0,004$ para no inferioridad) & $-2,45$ & $-2,24$ \\
\hline Episodios urgencia/24 h & $-2,85\left(^{*}\right)$ & $-2,42$ \\
\hline Episodios incontinencia de urgencia/24h & $-1,42(* *)$ & $-0,83$ \\
\hline Episodios incontinencia/24h & $-1,6(* *)$ & $-1,11$ \\
\hline Episodios nocturia/24h & $-0,71$ & $-0,63$ \\
\hline Reducción $50 \%$ episodios incontinencia & $74 \%(*)$ & $67 \%$ \\
\hline$\%$ Pacientes continentes & $59 \%(* *)$ & $49 \%$ \\
\hline Volumen miccional (cc) & $38\left(^{*}\right)$ & 31 \\
\hline № absorbentes/24h & $-1,72(* *)$ & $-1,19$ \\
\hline Percepción condición vesical (PPBC) & $-1,51(* *)$ & $-1,33$ \\
\hline
\end{tabular}

$\left({ }^{*}\left|p<0,05 ; 1^{* *}\right| p<0,01\right.$ 
TABLA VI. EFICACIA RESUMIDA DEL ESTUDIO SUNRISE (17).

\begin{tabular}{|l|c|c|c|}
\hline & Placebo & $\begin{array}{c}\text { Solifenacina } \\
\mathbf{5 - 1 0} \mathbf{~ m g}\end{array}$ & DES \\
\hline PPIUS (urgencia grados 3 y 4) & $-1,8$ & $-2,6$ & $\mathrm{P}<0,0001$ \\
\hline PPIUS (urgencia grados 1-4) & $-1,6$ & $-2,3$ & $\mathrm{P}<0,001$ \\
\hline № micciones / 24h & $-1,3$ & $-2,1$ & $\mathrm{P}<0,001$ \\
\hline Episodios incontinencia / 24h & $-1,4$ & $-1,7$ & $\mathrm{P}<0,001$ \\
\hline Episodios de incontinencia de urgencia /24h & $-1,3$ & $-1,7$ & $\mathrm{P}<0,001$ \\
\hline Molestia producida por la urgencia (VAS) & $35 \%$ & $59 \%$ & $\mathrm{P}<0,0001$ \\
\hline PPBC & $20 \%$ & $33 \%$ & $\mathrm{P}<0,0001$ \\
\hline Satisfacción con el tratamiento (VAS) & $34 \%$ & $66 \%$ & $\mathrm{P}<0,0001$ \\
\hline
\end{tabular}

cambios en la calidad de vida tras la introducción de un tratamiento son muy dispares entre el médico y el paciente, es necesario introducir instrumentos de medida para que el paciente cuantifique los cambios percibidos cuando investigamos nuevas terapias.

Entre los instrumentos de medida de parámetros subjetivos destacan el PPBC lesta escala ha sido recomendada como índice global único que puede complementarse con otros en la evaluación de tratamientos para la $\mathrm{VH}$ ), el cuestionario $\mathrm{OAB}-\mathrm{q}$ (instrumento multidimensional desarrollado para medir la autopercepción del paciente de sus síntomas de $\mathrm{VH}$ y el impacto que ésta genera sobre su calidad de vida, tanto en pacientes continentes como incontinentes; se compone de 33 preguntas en total, 8 relacionadas con la autopercepción de los síntomas en una escala de 8 puntos - desde me molesta nada hasta muchísimo-, y 25 relacionadas con la calidad de vida en una escala de 6 puntos -desde no me molestó en ningún momento hasta todo el tiempo-) y las escalas VAS (el paciente marca visualmente en una escala de $100 \mathrm{~mm}$ la intensidad de un síntoma percibido por sí mismo o la satisfacción con el tratamiento recibido, por ejemplo) (42).

El estudio SUNRISE (17) es un estudio europeo con 863 pacientes, en el que se compara solifenacina a dosis flexible de 5-10 mg frente al placebo durante 16 semanas de tratamiento. Se trata del primer estudio sobre $\mathrm{VH}$ donde la variable principal medida se refiere a la nueva definición de VH, y con- siste en el número de episodios de urgencia severa e incontinencia de urgencia definidos como urgencia de grado 3 y 4 en la escala PPIUS (Patient Perception of Intensity of Urgency Scale) (grado 3: urgencia severa, grado 4: urgencia que desencadena episodio de incontinencia). Las variables secundarias a estudio fueron la puntuación del cuestionario PPBC (Patient Perception of Bladder Condition), la molestia producida por la urgencia y la satisfacción con el tratamiento medida con escalas visuales VAS (Visual Analogue Scales), y todos los parámetros objetivos de los diarios miccionales ( $\mathrm{n}^{\circ}$ de episodios de urgencia/24h, $\mathrm{n}^{\circ}$ de micciones/24h, episodios de incontinencia/24h, episodios de incontinencia de urgencia/24h). La dosis flexible de solifenacina fue significativamente más eficaz que el placebo en la reducción de los episodios PPIUS grado 3-4 en 24 horas $(-2,6$ vs $-1,8$ con $P<0,001)$, lo que representa una reducción mediana del $70 \%$ respecto a los basales. Las mismas DES se dieron con todas las variables secundarias (Tabla VI). Un 47\% de los pacientes en tratamiento con solifenacina solicitaron aumento de dosis a las 8 semanas. Al igual que en el estudio anterior los pacientes que requirieron aumento de dosis son los que tenían basalmente síntomas más severos de $\mathrm{VH}(3)$.

\section{FASE IV (postcomercialización)}

El estudio VENUS $(43,44)$ es el homólogo del SUNRISE, realizado en USA con 739 pacientes y compara también solifenacina en dosis flexible con placebo durante 12 semanas. La variable principal 
TABLA VII. CAMBIO EN LAS VARIABLES TRAS EL TRATAMIENTO CON SOLIFENACINA EN EL ESTUDIO VENUS $(43,44)$.

\begin{tabular}{|l|c|c|}
\hline & Cambio tras tratamiento & P<0,0001 \\
\hline Episodios urgencia/24h & $-3,91$ (media) & $\mathrm{si}$ \\
\hline IUSS & $-0,9$ (media) & $\mathrm{si}$ \\
\hline UPS & $-0,4$ (media) & $\mathrm{si}$ \\
\hline Warning time $(\mathbf{s g})$ & 31,5 (mediana) & $\mathrm{P}<0,001$ \\
\hline OAB-q & 22,6 (media) & \\
\hline
\end{tabular}

estudiada fue el cambio en el número de episodios de urgencia en 24 horas registrados durante 3 días en un diario miccional. Como variables secundarias se midieron los cambios en el "warning time" y en las escalas de severidad de urgencia IUSS (Indevus Urgency Severity Scale) y de percepción de urgencia UPS (Urgency Perception Scale). Los pacientes con dosis flexible de solifenacina tuvieron DES en todos los parámetros medidos en cuanto a mejoría con respecto al placebo (Tabla VII).

El estudio VOLT (45) realizado en USA reclutó a 1743 pacientes evaluables en un estudio abierto de 12 semanas de duración con pacientes diagnosticados de $\mathrm{VH}$ que recibieron tratamiento con solifenacina $5 \mathrm{mg}$, pudiendo flexibilizar la dosis en las semanas 4 y 8 . Aproximadamente el $50 \%$ de los pacientes pidieron incrementar la dosis a $10 \mathrm{mg}$.
La particularidad es que además de recoger datos del diario miccional se obtuvieron datos subjetivos analizando la escala PPBC, la escala visual VAS y la percepción propia de los síntomas miccionales en el cuestionario OAB-q (Overactive Bladder Questionnaire). Se demostraron DES ( $p<0,001)$ al finalizar el estudio en la escala PPBC $(4,4$ vs. 2,9)(se pasó de percibir el problema vesical desde moderado-severo a pequeño-muy pequeño), en todas las subescalas que miden calidad de vida del cuestionario $O A B-q$ (cambios principales de 14,7 a 29,6 sobrepasando el límite de 10 puntos que se considera significativo) y en las escalas VAS (cambio medio de 36,9 vs. 41,8 en todos los síntomas medidos - urgencia, incontinencia de urgencia, frecuencia, nocturia) lo que indica una reducción significativa en la percepción por parte del paciente de los síntomas de $\mathrm{VH}$ (Tabla VIII).

TABLA VIII. RESULTADOS DE EFICACIA EN EL ESTUDIO VOLT (45).

\begin{tabular}{|l|c|c|c|c|}
\hline & Basal & Final & Cambio & DES \\
\hline Severidad síntomas OAB-q & 56,9 & 27,3 & $-29,6$ & $\mathrm{P}<0,001$ \\
\hline Escalas calidad vida OAB-q & 56,3 & 81,7 & $+25,4$ & $\mathrm{P}<0,001$ \\
\hline Urgencia (VAS) & 68,7 & 29,1 & $-39,6$ & $\mathrm{P}<0,001$ \\
\hline Incontinencia de urgencia (VAS) & 64,1 & 24 & $-40,1$ & $\mathrm{P}<0,001$ \\
\hline Frecuencia (VAS) & 70,6 & 28,8 & $-41,8$ & $\mathrm{P}<0,001$ \\
\hline Nocturia & 65,2 & 28,3 & $-36,9$ & $\mathrm{P}<0,001$ \\
\hline PPBC & 4,4 & 2,9 & $-1,5$ & $\mathrm{P}<0,001$ \\
\hline
\end{tabular}


Se realizó un subanálisis "post hoc" del estudio VOLT (46) donde los pacientes fueron estratificados según presentaban los síntomas que consideraban más molestos de su $\mathrm{VH}$, refiriendo basalmente el $27 \%$ la frecuencia, el $26 \%$ la incontinencia de urgencia, el $23 \%$ la urgencia y el $15 \%$ la nocturia. Se vio que las puntuaciones de las escalas VAS fueron peor en cada subgrupo concordante con el síntoma más molesto. Aunque todos los pacientes manifestaron mejoría en todas las escalas medidas (con DES $p<0,001$ ), los pacientes de cada subgrupo fueron los que refirieron una mayor mejoría al finalizar el estudio en esa escala VAS medida. Los pacientes del subgrupo "incontinencia de urgencia" fueron los que presentaron una mayor mejoría en las puntuaciones VAS, PPBC y escalas OAB-q.

El estudio VERSUS $(47,48)$ es un estudio prospectivo abierto de 12 semanas de duración con 441 pacientes, donde se comparó la eficacia de solifenacina en dosis flexible en pacientes que no experimentaron mejoría tras un tratamiento previo de al menos 4 semanas con tolterodina LP 4 mg (presentaban síntomas de urgencia residuales consistentes en 3 o más episodios de urgencia en 24 horas durante 3 días registrados en un diario miccional). La variable primaria de eficacia medida fue el cambio en el número de episodios de urgencia en 24 horas. Se midieron también variables de calidad de vida mediante el cuestionario OAB-q (33 preguntas), la percepción del paciente de su condición vesical (cuestionario PPBC), los cambios en la frecuencia miccional, nocturia e incontinencia de urgencia, la molestia que ocasionan los síntomas de $\mathrm{VH}$, los cambios en el estado de salud y CV relacionada con la salud con el cuestionario HUI 2/3 (Health Utilities Index Mark 2 and Mark 3), el uso de recursos médicos con el cuestionario MCUI (Medical Care Use Index) y el deterioro de la productividad en el trabajo o actividad diaria con el cuestionario WPAI-SHP (Work Productivity and Activity Impairment Questionnaire - Specific Health Problem). Los pacientes con solifenacina en dosis flexible tuvieron una reducción con DES $(p<0,0001)$ de todos los síntomas de $\mathrm{VH}$ en comparación con los que tomaban tolterodina LP antes del periodo de lavado ( $\mathrm{n}^{\circ}$ de episodios de urgencia, número de micciones, $\mathrm{n}^{\circ}$ de episodios de incontinencia y $\mathrm{n}^{\circ}$ episodios de nocturia en 24 horas). También se objetivó mejoría (DES $p<0,0001$ ) en todas las escalas del cuestionario OAB-q y en el PPBC. En el periodo de tratamiento con solifenacina se redujeron las visitas al médico, las infecciones urinarias y el uso de absorbentes con respecto al previo de tratamiento con tolterodina LP 4 $\mathrm{mg}$ (DES $p<0,0001)$. Lo mismo ocurrió con todos los parámetros medidos respecto a los cambios en la actividad diaria y la productividad laboral medidos con el WPAI-SHP. Los hallazgos de este estudio sugieren el beneficio al cambiar un antimuscarínico por otro en pacientes que no responden al primero. El 68,9\% de los pacientes en este estudio refieren una disminución en los problemas que les ocasiona su condición vesical tras dicho cambio (48) (Tabla IX).

El estudio VIBRANT (14) reclutó a 768 pacientes en USA con VH para analizar fundamentalmente cambios en la calidad de vida tras el tratamiento con solifenacina. El tratamiento fue con dosis flexible de $5-10 \mathrm{mg}$ durante 12 semanas. El estudio es pionero por utilizar como variable principal de eficacia el cambio en la puntuación de la escala de molestia sintomática del cuestionario $O A B-q$, donde se demostraron diferencias de 9,4 puntos con el grupo placebo $(p<0,0001)$. Secundariamente se objetivaron DES $(p<0,0001)$ en el $n^{\circ}$ de episodios de urgencia y frecuencia miccional /24 horas, el PPBC (que define la autopercepción del paciente de su condición vesical), el TS-VAS (que mide la satisfacción con el tratamiento), el BSW (define el beneficio, la satisfacción y la disposición a continuar con el tratamiento). En cuanto al WPAI (mide el absentismo laboral y la pérdida de productividad laboral asociados a su enfermedad) se objetivaron DES $(p<0,01)$. En el cuestionario ICIQ-MLUTSsex para medir los cambios relacionados con el tratamiento en la función sexual se vieron mejorías en las puntuaciones sin alcanzar DES. Como resumen podemos mencionar que el $84 \%$ de los pacientes reportó beneficios con el tratamiento activo, el $80 \%$ se encontró satisfecho y el $79 \%$ manifestó intención de continuar con éste (DES $p<0,0001$ frente a placebo).

Aunque disponemos de pocos estudios diseñados específicamente para comparar la eficacia entre diferentes antimuscarínicos, existe un análisis con 7 estudios controlados con placebo donde la disminución media (y mediana) de los episodios de urgencia fue mayor con solifenacina que con otros como oxibutinina, tolterodina, trospio, o propiverina (49).

\section{Eficacia en términos de calidad de vida}

Los síntomas de $\mathrm{VH}$ producen en las personas que los sufren un impacto negativo en su calidad de vida, ocasionando vergüenza, frustración, ansiedad, depresión y aislamiento social $(50,51,52,53)$. Aunque clásicamente se han medido parámetros objetivos en los ensayos clínicos para valorar la eficacia del fármaco a estudio actualmente la tendencia es a introducir y dar igual valor a una serie de parámetros de medición subjetivos, entre ellos la calidad de vida mediante diferentes instrumentos.

La ICS recomienda medir la calidad de vida en todos los estudios que evalúen los distintos trata- 
mientos para la $\mathrm{VH}$ como complemento a los instrumentos que miden los síntomas o su intensidad. Los cuestionarios de calidad de vida cuantifican las molestias que estos síntomas ocasionan al paciente y su mejoría tras un tratamiento eficaz. Son un medidor indirecto de la eficacia de un fármaco y de la satisfacción del paciente con el tratamiento, indicándonos cual es el impacto que ocasiona la $\mathrm{VH}$ en la vida del sujeto (54).

Una herramienta muy utilizada es el King's Health Questionnaire (KHQ), un cuestionario corto, sensible y específico para $\mathrm{VH}$ donde se miden 10 escalas relacionadas con la vida y salud del paciente.

En los estudios de fase llla de 12 semanas de duración con una extensión de 40 semanas $(15,23$,
$24,54)$ también se utilizó el KHQ. Tanto en el grupo de solifenacina $5 \mathrm{mg}$ como $10 \mathrm{mg}$ se vieron diferencias significativas en 9 de las 10 escalas medidas, que además se correlacionan con los cambios en los síntomas de VH (22). En uno de los estudios a 12 semanas con 1033 pacientes se demostraron DES a dosis de 5 y $10 \mathrm{mg}$ en 5 de las 10 escalas del cuestionario, y en cuanto al impacto de la incontinencia también se apreciaron DES con $10 \mathrm{mg}$. En el segundo de ellos con 857 pacientes se encontraron DES en las escalas de impacto de la incontinencia, limitaciones sociales, emociones, sueño/energía, y severidad de los síntomas para 5 y $10 \mathrm{mg}$. También en las limitaciones físicas y en la medida de la severidad a 10 $\mathrm{mg}$ se encontraron DES. Uniendo y analizando los datos conjuntamente se vieron DES con solifenacina en 9 de las 10 escalas medidas con el KHQ (54).

TABLA IX. RESULTADOS DE EFICACIA EN ESTUDIO VERSUS $(47,48)$.

\begin{tabular}{|l|c|c|c|}
\hline & Cambio & $\begin{array}{c}\text { \% cambio } \\
\text { (mediana) }\end{array}$ & DES \\
\hline № episodios urgencia / 24h & $-4,2$ & $-75 \%$ & $\mathrm{P}<0,001$ \\
\hline № micciones / 24h & $-2,3$ & $-19 \%$ & $\mathrm{P}<0,001$ \\
\hline № episodios incontinencia /24h & $-2,6$ & $-100 \%$ & $\mathrm{P}<0,001$ \\
\hline № episodios nocturia / 24h & $-0,8$ & $-43,7 \%$ & $\mathrm{P}<0,001$ \\
\hline Molestias sintomáticas (OAB-q) & $-29,5$ & $-51,5 \%$ & $\mathrm{P}<0,001$ \\
\hline Escalas calidad de vida (OAB-q) & $+25,2$ & $+44,4 \%$ & $\mathrm{P}<0,001$ \\
\hline PPBC & $-1,2$ & & $\mathrm{P}<0,001$ \\
\hline № visitas al médico & -1 & & $\mathrm{P}<0,0001$ \\
\hline № infecciones urinarias & $-0,12$ & & $\mathrm{P}<0,0001$ \\
\hline № lesiones cutáneas & $-0,46$ & & $\mathrm{NDS}$ \\
\hline № caídas & 0,04 & & $\mathrm{P}$ \\
\hline № absorbentes utilizados & $-2,79$ & & $\mathrm{P}<0,0001$ \\
\hline \% tiempo de trabajo perdido & $-1,84$ & & \\
\hline \% tiempo perdido durante el trabajo & $-11,6$ & & \\
\hline \% tiempo perdido de actividades & & & \\
\hline
\end{tabular}

NDS: no diferencias estadísticamente significativas. 
En el estudio de extensión con 1347 pacientes analizados se menciona una mejoría del 17\% referida en la percepción del estado de salud y un $35-48 \%$ para los otros dominios. El incremento de mejoría global en las escalas medidas desde que finalizaron los estudios de 12 semanas hasta el final de las 52 de la extensión fue estimado en un $28-35 \%$ (54).

Cuando los datos de calidad de vida se comparan con los de eficacia en estos estudios vemos curiosidades como la relación en la mejoría de la escala que mide el impacto de la incontinencia con la disminución de los episodios de incontinencia, la relación en la mejoría de la escala sueño/energía con la disminución de los episodios de nocturia, y cómo la satisfacción con la eficacia percibida por el paciente asimismo se relaciona con el avance del estudio $(48 \%$ a las 4 semanas, $68 \%$ a las 16 semanas, $74 \%$ a las 28 semanas) al mismo tiempo que la mejoría en calidad de vida demostrada en el estudio de extensión $(15,54)$.

En el estudio VOLT (45) se comienza a utilizar el cuestionario OAB-q, que incluye 25 escalas para medir calidad de vida y cómo afectan estos síntomas a sus vidas. Se considera que para objetivar diferencias significativas debe haber una diferencia de 10 puntos tras la intervención en todas las escalas medidas. En dicho estudio se vio mejoría con DES $(p<0,001)$ en todas las escalas tras el tratamiento con solifenacina, correlacionándose con la mejoría en todas las escalas VAS de percepción de síntomas de $\mathrm{VH}$ (urgencia, frecuencia, nocturia e incontinencia de urgencia). Se apreciaron DES en todos los dominios excepto para el "sueño", viendo las diferencias más importantes en el subgrupo de pacientes con "nocturia" (46). Durante el estudio VERSUS (47) se volvió a comprobar que los pacientes en el grupo de solifenacina tenían una mejoría con DES ( $p<0,001)$ tanto en las escalas de calidad de vida como de molestias percibidas de los síntomas medidas con el cuestionario $\mathrm{OAB}-\mathrm{q}$.

El estudio VIBRANT es el primero conocido que fue diseñado para apreciar cambios en los parámetros de calidad de vida como variables primarias de eficacia mediante el cuestionario OAB-q. Los datos son consistentes con todos los estudios anteriores viendo mejoría con DES desde las 4 semanas de tratamiento con solifenacina, aumentando las diferencias hasta las 12 semanas (14).

\section{Satisfacción y adherencia al tratamiento}

Se ha observado que el hecho de flexibilizar la dosis hace que aumente la satisfacción del paciente con el tratamiento, donde tiene opción de participar en el ajuste de su dosis eficaz para reducir al mínimo los efectos secundarios.
Un signo indirecto para medir la satisfacción con el tratamiento es la cuantificación de la adherencia al tratamiento. En todos los estudios en fase III las pérdidas de pacientes por efectos adversos fueron insignificantes y comparables al grupo placebo (23, $24,41)$. El $91 \%$ de pacientes que completaron 12 semanas de tratamiento aceptaron continuar en tratamiento durante 40 semanas lo cual es indicativo de una alta satisfacción con el tratamiento. De ellos el $81 \%$ completaron la extensión indicando asimismo que la satisfacción se mantiene a largo plazo.

En el estudio de extensión de solifenacina (15) el 99\% de los pacientes cuando se les preguntó sobre la tolerabilidad contestaron que fue satisfactoria o aceptable. El $74 \%$ al finalizar el estudio manifestaron satisfacción con la eficacia.

\section{CONCLUSIONES}

Como sabemos en la actualidad el síntoma "urgencia" es el que define el síndrome de VH, y es considerado el síntoma "gatillo" que desencadena el resto, por lo tanto, debería se el objetivo central en el tratamiento. Los síntomas de llenado vesical tienen un severo impacto en todas las facetas de la calidad de vida de una persona, incluidas la social, psicológica y laboral. El tratamiento de una enfermedad crónica que no tiene riesgo vital como la $\mathrm{VH}$ se debe centrar en el beneficio del paciente y en la mejoría autopercibida en su calidad de vida más que en la resolución de los síntomas objetivos (aunque ambos estén interrelacionados). Es preciso un balance entre eficacia y tolerabilidad para que los beneficios sean palpables y promuevan la persistencia terapéutica. La VH produce en los pacientes un importante impacto negativo en su calidad de vida ocasionando vergüenza, ansiedad, depresión y puede desencadenar aislamiento social. En la actualidad la ICS recomienda la medición de parámetros de calidad de vida en todos los estudios que evalúen tratamientos para la $\mathrm{VH}$. Constituyen un indicador indirecto de eficacia y satisfacción que puede considerarse igual o más importante que los objetivos.

En el resultado del tratamiento de pacientes con $\mathrm{VH}$ es muy importante valorar las expectativas que el paciente tiene de dicho tratamiento, ya que normalmente el paciente tiende a sobrevalorar su efectividad, por tanto, el médico debe aclarar los límites tanto en la disminución de síntomas como de aparición de efectos secundarios. Los pacientes que experimentan precozmente efectos secundarios son más reacios a finalizar el tratamiento, como ocurre cuando se comienza con la dosis mayor. La flexibilización de dosis es una estrategia que permite utilizar 
los antimuscarínicos de la forma más costo-efectiva ofreciendo la dosis mayor para aquellos pacientes que lo necesiten.

Como demuestran la mayoría de estudios, la dosis flexible de solifenacina mejora los síntomas de $\mathrm{VH}$ medidos tanto de forma objetiva como subjetiva. El aumento de dosis proporciona un aumento extra de eficacia como vemos en los estudios STAR y SUNRISE. En la mayoría de estudios con flexibilización de dosis apreciamos que aproximadamente el $50 \%$ de los pacientes cuando se les pregunta piden aumentar la dosis de 5 a $10 \mathrm{mg}$ de solifenacina porque creen que podrían mejorar aún más sus síntomas. Este grupo coincide con los que presentaban basalmente síntomas más severos o provenían de un tratamiento previo no efectivo. En la mayoría de estudios asimismo se aprecia un incremento adicional de eficacia aumentando esta dosis. Existe un estudio de 12 semanas de 4450 pacientes con comorbilidades y comedicación en Alemania donde la cifra de pacientes tomando crónicamente $10 \mathrm{mg}$ asciende al $21 \%(29)$.

El efecto placebo en todos los ensayos clínicos que investigan síntomas del tracto urinario inferior no es despreciable, pudiendo alcanzar hasta el 15-30\% (Tabla III). Se ha mencionado que el paciente que rellena diarios miccionales tiene más presente sus hábitos miccionales y podría sobreestimar la eficacia de los tratamientos. También puede contribuir a ésto el mayor apoyo médico y de enfermería que sufren durante dichos estudios. Por eso, hay que ser especialmente exquisitos en el diseño de los estudios y utilizar siempre una rama de pacientes con placebo como comparador. Se ha sugerido por todo lo anterior, que en ocasiones pequeñas diferencias estadísticamente significativas entre los grupos de placebo y de tratamiento activo pueden suponer una diferencia considerable en la calidad de vida y en la percepción del paciente de mejoría y satisfacción (55). Todo ello refuerza la idea de medir parámetros subjetivos y de autopercepción del problema vesical en este tipo de estudios.

La eficacia de la solifenacina en el tratamiento de la VH ha sido sobradamente probada frente al placebo y algún antimuscarínico en estudios de fase II, III y IV. En un primer tiempo se demostraron DES cuando se medían parámetros objetivos miccionales. Más adelante el diseño de los estudios sufrió un cambio incorporando multitud de instrumentos para medir síntomas subjetivos y autopercibidos por el paciente, así como para medir molestias sintomáticas y calidad de vida. Con estos nuevos parámetros se demostró que los síntomas objetivos y subjetivos estaban correlacionados, viéndose que si mejoran los datos de los diarios miccionales también lo hacen en consonancia los datos de calidad de vida y autopercepción del problema vesical.

La Solifenacina es eficaz para el control de los episodios de urgencia miccional, reduciéndolos de forma significativa en el $60 \%$ de los casos. Su papel en el aumento del "warning time" hace aumentar la capacidad funcional de la vejiga y el volumen de cada micción, reduciendo consecuentemente la frecuencia, la nocturia y la incontinencia. La eficacia de solifenacina se confirma no solo a corto plazo sino también a largo plazo, viéndose en el estudio a largo plazo (52 semanas) una mejoría en todos los síntomas principales (la urgencia un 63\%, la frecuencia miccional un $23 \%$, la incontinencia un $66 \%$ $y$ el volumen miccional un $31 \%$ ). Al final del estudio el $40 \%$ de los pacientes no reportaron urgencia y el $58 \%$ habían recuperado la continencia (15).

Los pacientes del subgrupo con incontinencia de urgencia fueron los que presentaron proporcionalmente una mejoría mayor en las puntuaciones de variables como autopercepción de la condición vesical, escalas sintomáticas y de calidad de vida en el OAB-q, y escalas VAS de molestias sintomáticas / satisfacción con el tratamiento (46). Se ha demostrado beneficio al cambiar un antimuscarínico cuando falla otro. En concreto, en el estudio VERSUS se demostró que el $69 \%$ de los pacientes refirieron mejorar su condición vesical con solifenacina tras el fracaso con tolterodina, incluso se redujeron las visitas médicas, las infecciones urinarias y el uso de absorbentes, aumentando la productividad laboral y la actividad cotidiana.

Si nos centramos en la medición de calidad de vida como parámetro fundamental de eficacia (cambio en las escalas del OAB-q), apreciamos que el $84 \%$ de los pacientes tiene beneficios con solifenacina, el $80 \%$ se encuentra satisfecho al finalizar el tratamiento y el $79 \%$ manifiesta su intención de continuar con éste (14).

Si analizamos aisladamente el uso de $10 \mathrm{mg}$ de solifenacina en 4 estudios de fase III con 2800 pacientes (41), vemos una reducción de los episodios de urgencia / $24 \mathrm{~h}$ del $70 \%$, de la frecuencia miccional del $23 \%$, de los episodios de nocturia / $24 \mathrm{~h}$ del $36 \%$, de los episodios de incontinencia / $24 \mathrm{~h}$ del $100 \%$ y un aumento del volumen miccional medio del $26 \%$ (DES $p<0,001)$. Un 51,8\% se vuelven continentes (DES $p<0,001$ ).

Con Solifenacina apenas se han descrito efectos adversos graves, siendo valorados como leves o moderados en la mayoría de los casos. La posi- 
bilidad de flexibilizar la dosis permite individualizar en cada paciente con su participación la dosis más efectiva y tolerable. En los estudios al efecto no se ha demostrado un aumento significativo de efectos adversos al subir la dosis de 5 a $10 \mathrm{mg}$, corroborándose en el estudio de extensión con 52 semanas de tratamiento, donde el $91 \%$ estuvo dispuesto a continuar 40 semanas más, al final de éste el $85 \%$ de los pacientes se manifiesta satisfecho con la tolerabilidad del tratamiento, y el $99 \%$ refieren una tolerabilidad satisfactoria o aceptable. Siendo la sequedad de boca el efecto adverso más frecuente, más del $92 \%$ lo refiere como leve-moderado, y tan sólo el $1,4-4,7 \%$ de los pacientes en ensayos clínicos abandonan éstos por efectos adversos, sin diferencias significativas frente al placebo. El estreñimiento no suele ser un problema relevante salvo en población anciana con síntomas previos. La visión borrosa presenta unas cifras por debajo del $3 \%$ con el régimen de dosis flexible y en su mayoría de características leves-moderadas. El resto pueden enmarcarse en el rango de lo infrecuente $(<1 \%)$. En general, los efectos secundarios fueron superponibles entre poblaciones de jóvenes y ancianos.

\section{BIBLIOGRAFIA y LECTURAS RECOMENDADAS (*lectura de interés $y^{* *}$ lectura fundamental)}

**1. Abrams P, Cardozo L, Fall M, Griffiths D, Rosier P, Ulmsten U et al. The standardisation of terminology of lower urinary tract function: report from the standardisation sub-committee of the International Continence Society. Neurourol Urodyn 2002; 21:167-78.

2. Abrams P, Artibani W, Cardozo L, Dmochowski R, van Kerrebroeck P, Sand P. Reviewing the ICS 2002 terminology report: the ongoing debate. Neurourol Urodyn 2009; 28(4): 293.

3. Chapple CR, Rosenberg MT, Brenes FJ. Listening to the patient: a flexible approach to the use of antimuscarinic agents in overactive bladder syndrome. BJU Int 2009; 104(7): 960-7.

*4. Irwin DE, Milsom I, Hunskaar S et al. Populationbased survey of urinary incontinence, overactive bladder and other lower urinary tract symptoms in five countries: results of the EPIC study. Eur Urol 2006; 50: 1306-15.

*5. Milsom I, Abrams P, Cardozo L, Roberts RG, Thüroff J, Wein AJ. How widespread are the symptoms of an overactive bladder and how are they managed? A population-based prevalence study. BJU Int 2001; 87: 760-6.
*6. Stewart WF, Van Rooyen JB, Cundiff GW, Abrams, P.; Herzog, A.R.; Corey, R, et al. Prevalence and burden of overactive bladder in the United States. World J Urol 2003; 20: 327-36.

7. Robinson, D.; Cardozo, L. Solifenacin: pharmacology and clinical efficacy. Expert Rev Clin Pharmacol 2009; 2(3): 239-253.

8. Wagner TH, Hu TW, Bentkover J, Le Blanc, K.; Stewart, W.; Corey, R, et al. Health-related consequences of overactive bladder. Am J Manag Care 2002; 8(19 suppl.):S598-S607.

9. Hu TW, Wagner TH. Health-related consequences of overactive bladder: an economic perspective. BJU Int 2005; 96(1): 43-5.

10. Nabi G, Cody JD, Ellis G, Herbison P, Hay-Smith J. Anticholinergics drugs versus placebo for overactive bladder syndrome in adults. Cochrane Database Syst. Rev. 4 2006, CD003781.

**11. Ohtake A, Ukai M, Hatanaka T, Kobayashi, S, Ikeda, K, Sato, S, et al. In vitro and in vivo tissue selectivity profile of solifenacin succinate (YM905) for urinary bladder over salivary gland in rats. Eur J Pharmacol 2004; 492: 243-50.

12. Chapple, C.R. Solifenacin provides effective antimuscarinic therapy for the complete management of overactive bladder. Expert Opin Pharmacother 2006; 7(17): 2421-34.

13. Ficha Técnica Vesicare ${ }^{\circledR} 5$ y $10 \mathrm{mg}$.

**14. Vardy M.D, Mitcheson, H.D, Samuels, T.A, Wengeke, J.D, Forero-Schwanhaeuser, S, Marshall, T.S et al. Effects of solifenacin on overactive bladder symptoms, symptom bother and other patient-reported outcomes: results from VIBRANT - a double-blind, placebo-controlled trial. Int $\mathrm{J}$ Clin Pract 2009; 63(12): 1702-14.

**15. Haab F, Cardozo L, Chapple C, Ridder, A.M. Long-term open label solifenacin treatment associated with persistence with therapy in patients with overactive bladder syndrome. Eur Urol 2005;47:376-84.

**16. Chapple CR, Martinez-Garcia R, Selvaggi L, Toozs-Hobson, P.; Warnack, W.; Drogendijk, T et al. A comparison of the efficacy and tolerability of solifenacin succinate and extended release tolterodine at treating overactive bladder syndrome: results of the STAR trial. Eur Urol 2005;48(3):46470.

**17. Cardozo L, Hessdoerfer, E, Milani, R, Araño, P, Dewilde, L, Slack, M, et al. Solifenacin in the treatment of urgency and other symptoms of overactive bladder: results from a randomized, double-blind, placebo-controlled, rising-dose trial. BJU Int 2008; 102(9): 1120-7.

18. Appell RA. Recent clinical studies of new pharmacologic agents and their efficacy in the treatment of incontinence. Rev Urol 2001; 3 (Suppl 1): $\mathrm{S} 15-8$. 
19. Zinner N, Gittleman M, Harris R, Susset J, Kanelos A, Auerbach S. R et al. Trospiun chloride improves overactive bladder symptoms: a multicenter Phase III trial. J Urol 2004; 171(6 Pr 1): 2311-15.

20. Abrams P, Cardozo L, Chapple CR, Serdarevic, D, Hargreaves, K, Khullar, V. Comparison of the efficacy, safety and tolerability of propiverine and oxybutinin for the treatment of overactive bladder syndrome. Int J Urol 2006; 13(6): 692-8.

21. Nitti VW, Dmochowski R, Appel RA, Wang, J.T, Bavendam, T, Guan, Z.H. Efficacy and tolerability of tolterodine extended-release in continent patients with overactive bladder and nocturia. BJU Int 2006; 97(6): 1262-6.

22. Payne CK. Solifenacin in overactive bladder syndrome. Drugs 2006; 66(2): 175-90.

**23. Chapple CR, Rechberger T, Al-Shukri S, Meffan, P, Everaert, K, Huang, M, et al. Randomized, double-blind placebo controlled and tolterodine controlled trial of the once daily antimuscarinic agent solifenacin in patients with symptomatic overactive badder. BJU Int 2004; 93(3):303-10.

**24. Cardozo L, Lisec M, Millard R, van Vierssen Trip, O, Kuzmin, I, Drogendijk T.E, et al. Randomized, double-blind placebo controlled trial of the once daily antimuscarinic agent solifenacin succinate in patients with overactive bladder. J Urol 2004;172:1919-24.

**25. Chu F, Smith N, Uchida T. Efficacy and safety of solifenacin succinate $10 \mathrm{mg}$ once daily: a multicenter, phase III, randomized, double-blind, placebo-controlled, parallel-group trial in patients with overactive bladder. Current Ther Res 2009; 70 (6): 405-20.

26. Dmochowski R, Staskin, D.R . The Q-T interval and antimuscarinic drugs. Curr Urol Rep 2005;6(6):405-9

27. Krauwinkel WJ, Smulders RA, Mulder H, Swart PJ, Taekema-Roelvink ME. Effect of age on the phamacokinetics of solifenacin in men and women. Int J Clin Pharmacol Ther 2005; 43(5): 227-38.

*28. Wagg A, Wyndaele JJ, Sieber P. Efficacy and tolerability of solifenacin in elderly subjects with overactive bladder syndrome: a pooled analysis. Am J Geriatr Pharmacother 2006; 4(1): 14-24.

*29. Michel, M C, Wetterauer U, Vogel M, de la Rosette J J M H. Cardiovascular safety and overall tolerability of solifenacin in routine clinical use. Drug Safety 2008;31(6):505-14.

*30. Wesnes KA, Edgar C, Tretter R, Bolodeoku J. Exploratory pilot study assessing the risk of cognitive impairment or sedation in the elderly following single doses of solifenacin $10 \mathrm{mg}$. Expert Opin Drug Saf 2009; 8(6): 615-26.

31. Swart PJ, Krauwinkel WJ, Smulders RA, Smith NN. Pharmacokinetic effect of ketoconazole on solifenacin in healthy volunteers. Basic Clin Pharmacol Toxicol 2006; 99: 33-6.

32. Taekema-Roelvink ME, Swart PJ, Kuipers ME, Krauwinkel WJ, Visser N, Smulders RA. Pharmacokinetic interaction of solifenacin with an oral contraceptive containing ethinyl estradiol and levonorgestrel in healthy women: a double blind, placebo controlled study. Clin Ther 2005; 27: 1403-10.

33. Smulders RA, Kuipers MA, Krauwinkel WJJ. Multiple doses of the antimuscarinic agent solifenacin do not affect the phamacodynamics or pharmacokinetics of warfarin of the steady-state pharmacokinetics of digoxin in healthy subjects. Br J Clin Pharmacol 2006; 62: 210-7.

34. Smulders RA, Smith NN, Krauwinkel WJ, Hoon TJ. Phamacokinetics, safety and tolerability of solifenacin in patient with renal insufficiency. $\mathbf{J}$. Pharmacol Sci 2007; 103: 67-74.

35. Kuipers M, Smulders R, Krauwinkel W, Hoon T. Open label study of the safety and pharmacokinetics of solifenacin in subjects with hepatic impairment. J Phamacol Sci 2006; 102: 405-12.

**36. Chapple CR, Araño P, Bosch JL, De Ridder D, Kramer AE, Ridder AM. Solifenacin appears effective and well tolerated in patients with symptomatic idiopathic detrusor overactivity in a placebo and tolterodine controlled Phase II dose finding study. BJU Int 2004; 93(1): 71-7.

37. Chapple CR, Araño P, Bosch JLHR, De Ridder D, Kramer G, Ridder AM. YM905 appears effective and well tolerated in patients with symptomatic idiophatic detrusor overactivity in an european placebo and tolterodine controlled Phase II dose finding study. Neurourol Urodyn 2002; 21 (4): 381-2.

38. Gittleman MC. The efficacy and safety of solifenacin in adults with overactive bladder; a multicentre, placebo controlled study. Int J Gynaecol Obstet 2003; 83 (Suppl 3): 94.

39. Gittelman, M.; Chu, F.M.; Klimberg, I.; Fincher, R.; Smith, N.; Tempel, D et al. Two randomized, double-blind, placebo-controlled, parallel-group, fixed- dose, multicenter studies assess the efficacy and safety of daily oral administration of $10 \mathrm{mg}$ YM905 versus placebo in male and female subjects with overactive bladder. J Urol 2003 169(4 Suppl): 349

40. Cardozo L, Chapple C, Steers W, Govier F. Solifenacin improves all symptoms of overactive bladder syndrome. Neurourol Urodyn 2005; 24(56): Abstr. 300

41. Chapple CR, Cardozo L, Steers WD, Govier FE. Solifenacin significantly improves all symptoms of overactive bladder syndrome. Int J Clin Pract 2006; 60(8): 959-66.

42. Matza LS, Thompson CL, Krasnow J, Brewster-Jordan J, Zyczynski T, Coyne KS et al. Test- 
retest realiability of four questionnaires for patients with overactive bladder: The overactive bladder questionnaire (OAB-q), patient perception of bladder condition (PPBC), urgency questionnaire (UQ), and the primary OAB symptom questionnaire (POSQ). Neurourol Urodyn 2005; 24: 215-25.

**43. Karram MM, Toglia MR, Serels SR, Andoh M, Fakhoury A, Forero-Schwanhaeuser S. Treatment with solifenacin increases warning time and improves symptoms of overactive bladder: results from VENUS, a randomized, doubleblind, placebo-controlled trial. Urology 2009; 73(1): 14-18.

*44. Toglia MR, Serels SR, Laramee C, Karma MM, Nandy IM, Andoh M, et al. Solifenacin for overactive bladder: patient-reported outcomes from a large placebo-controlled trial. Posgraduate Med 2009; 121(5): 151-8.

**45. Garely AD, Kaufman JM, Sand PK, Smith N, Andoh M. Symptom bother and health-related quality of life outcomes following solifenacin treatment for overactive bladder: the VESIcare Open-Label Trial. Clin Ther 2006; 28(11):1935-1946.

*46. Sand PK, Steers WD, Dmochowski R, Andoh M, Forero-Schwanhaeuser S. Patient-reported most bothersome symptoms in OAB: post hoc analysis of data from a large, open-label trial of solifenacin. Int Urogynecol J Pelvic Floor Dysfunct. 2009; 20(6):667-75.

*47. Zinner N, Noe L, Rasouliyan L, Marshall T, Seifeldin R. Impact of solifenacin on resource utilization, work productivity and health utility in overactive bladder patients switching from tolterodine ER. Curr Med Res Opin 2008; 24(6): 1583-91.

**48. Chancellor MB, Zinner N, Whitmore K, Kobashi K, Snyder JA, Siami, P et al. Efficacy of solifenacin in patients previously treated with tolterodine extended release $4 \mathrm{mg}$ : results of a 12-week, multicenter, open-label, flexible-dose study: Clin Ther 2008; 30(10):1766-81.

49. Michel MC, de la Rosette JJMCH. Role of muscarinic receptor antagonist in urgency and nocturia. BJU Int 2005; 96(Suppl. 1): 37-42.

50. Debruyne FM, Heesakkers JP. Clinical and socioeconomic relevance of overactive bladder. Urology 2004; 63 (3 Suppl. 1): 42-4.

51. Epstein LB, Goldberg RP. The overactive bladder and quality of life. Int J Fertil Women Med 2005; 50(1): 30-6.

52. Mullins CD, Subak LL. New perspectives on overactive bladder: quality of life impact, medication persistency, and treatment costs. Am J Manag Care 2005; 1(4 Suppl.): S101-2.

53. Hashim H, Abrams P. Drug treatment of overactive bladder: efficacy, cost and quality of life consideration. Drugs 2004; 64(15): 1643-56.

*54. Kelleher CJ, Cardozo L, Chapple CR, Haab F, Ridder AM. Improved quality of life in patients with overactive bladder symptoms treated with solifenacin. BJU Int 2005; 95:81-5.

55. van Leeuwen JHS, Castro R, Busse M, Bemelmans BLH. The placebo effect in the pharmacologic treatment of patients with lower urinary tract symptoms. Eur Urol 2006. 50: 440-53. 